\title{
Growth Pattern and Prognostic Factors of Untreated Nonfunctioning Pituitary Adenomas
}

\author{
Kihwan Hwang, M.D., ${ }^{1}$ Taehun Kwon, M.D., ${ }^{2}$ Jay Park, M.D., ${ }^{3}$ Jin-Deok Joo, M.D., ${ }^{4}$ Jung Ho Han, M.D., \\ Chang Wan Oh, M.D., Ph.D., ${ }^{1,2}$ Chae-Yong Kim, M.D., Ph.D. ${ }^{1,2}$ \\ Department of Neurosurgery, Seoul National University Bundang Hospital, Seongnam, Korea \\ Department of Neurosurgery, ${ }^{2}$ Seoul National University College of Medicine, Seoul, Korea \\ Department of Neurosurgery, ${ }^{3}$ The Univerisity of Edinburgh Edinburgh Medical School, Edinburgh, UK \\ Department of Neurosurgery, ${ }^{4}$ Jeju National University Hospital, Jeju, Korea
}

Objective : Pituitary adenomas (PAs) are often detected as incidental findings. However, the natural history remains unclear. The objective of this study was to evaluate the natural history and growth pattern of untreated PAs.

Methods : Between 2003 and 2014, 59 PAs were managed with clinico-radiological follow up for longer than 12 months without any kind of therapeutic intervention. Tumor volumes were calculated at initial and last follow-up visit, and tumor growth during the observation period was determined. Data were analyzed according to clinical and imaging characteristics.

Results : The mean initial and last tumor volume and diameter were $1.83 \pm 2.97 \mathrm{~mL}$ and $13.77 \pm 6.45 \mathrm{~mm}, 2.85 \pm 4.47 \mathrm{~mL}$ and $15.75 \pm 8.08 \mathrm{~mm}$, respectively. The mean annual tumor growth rate was $0.33 \pm 0.68 \mathrm{~mL} /$ year during a mean observation period of 46.8 \pm 32.1 months. Sixteen (27\%) PAs showed tumor growth. The initial tumor size (HR, 1.140; 95\% confidence interval, 1.003-1.295; $p=0.045$ ) was the independent predictive factor that determined the tumor growth. Six patients $(11 \%)$ of 56 conservatively managed non-symptomatic PAs underwent resection for aggravating visual symptoms with mean interval of 34.5 months from diagnosis. By Cox regression analysis, PAs of last longest diameter over $21.75 \mathrm{~mm}$ were a significant prognostic factor for eventual treatment.

Conclusion : The initial tumor size of PAs was independently associated with the tumor growth. Six patients (11\%) of conservatively managed PAs were likely to be treated eventually. PAs of last follow-up longest diameter over $21.75 \mathrm{~mm}$ were a significant prognostic factor for treatment. Further studies with a large series are required to determine treatment strategy.

Key Words : Pituitary adenoma · Observation $\cdot$ Natural history $\cdot$ Volumetric analysis $\cdot$ Prognosis.

\section{INTRODUCTION}

It was in 1936 when Costello first described pituitary inci- dentalomas (PIs) that they occurred with the remarkable frequency of $22.5 \%{ }^{4}$. Since then, his investigation has been cited and reexamined in numerous publications. The prevalence of

• Received : July 16, 2018 •Revised : September 19, 2018 •Accepted : September 27, 2018

- Address for reprints : Chae-Yong Kim, M.D., Ph.D.

Department of Neurosurgery, Seoul National University Bundang Hospital, 82 Gumi-ro 173beon-gil, Bundang-gu, Seongnam 13620, Korea

Tel : +82-31-787-7165, Fax : +82-31-787-4059, E-mail : chaeyong@snu.ac.kr

This is an Open Access article distributed under the terms of the Creative Commons Attribution Non-Commercial License (http://creativecommons.org/licenses/by-nc/4.0) which permits unrestricted non-commercial use, distribution, and reproduction in any medium, provided the original work is properly cited. 
PIs in autopsy series is known to be around 3-27\%,3,18,20) and on imaging studies around $10 \%{ }^{10,12,17)}$. Although, PIs are common in clinical practice, little is known about the long-term natural history.

Pituitary adenomas (PAs) are not usually associated with clinical syndromes related to hormone excess and may be discovered occasionally, when they are not hormonally active ${ }^{13}$. So, they usually escape early diagnosis and are mainly discovered when they exert mass effects on surrounding tissues leading to visual impairments, headache and hypopituitarism ${ }^{9}$. The option for treatment for patients with symptomatic PAs is debulking surgery, but, in the absence of mass effects, a "wait and see" approach with a serial evaluation of tumor growth and visual function could be a possible therapeutic choice ${ }^{8)}$. However, patient series systematically reporting the PAs outcome that were not treated either surgically, medically, or with radiotherapy during long-term follow-ups are scarce ${ }^{8,13)}$.

The aim of this study was to evaluate the natural history and growth pattern of untreated nonfunctioning PAs, as well as to assess clinico-radiologic prognostic factors, using 3-dimensional volumetric analysis. Proper understanding of these features is imperative to guide treatment consensus for patients with PAs.

\section{MATERIALS AND METHODS}

\section{Patient selection}

This was a retrospective cohort study of patients with PAs under observation without any therapeutic intervention. Between July 2003 and December 2014, 855 patients were newly diagnosed as PAs at Seoul National University Bundang Hospital. This study was approved by the ethical committee of authors' institution (IRB No. B-1807/483-105). Our inclusion criteria of this study were as follows : 1) magnetic resonance imaging (MRI) definitely suggestive of PA, neither Rathke's cleft cyst nor other sellar tumors; 2) at least two sequential radiological follow-ups over a minimum interval of 12 months; 3) at least one complete clinical and biochemical assessment; and 4) conservative management without any intervention. Of a total population, 498 patients (58\%) were placed in a treatment group immediately after diagnosis and 298 patients (35\%) were with either inappropriate medical records or absence of follow up images. Excluding these patients, the re- maining 59 patients (7\%) were managed with clinico-radiological follow-up for longer than 12 months without any kind of therapeutic intervention (Fig. 1). They were observed with a "wait-and-see" policy and included as final analysis.

\section{Volumetric measurements}

Tumor size was measured volumetrically using the initial and the last follow-up MRI, and tumor growth during the observation period was determined. Using a stereological method according to the Cavalieri principle, the three-dimensional tumor volumes were calculated from two-dimensional imag$\mathrm{es}^{16)}$. The volume of the tumor was estimated from the volumetric measurement of the coronal plane Gadolinium-enhanced T1-weighted MRI with the slice thickness of $2 \mathrm{~mm}$. The tumor area was figured out by tracing its contour on each MRI slices using the Picture Archiving and Communication System (version 3.0.9.1 BN 13, 2005; INFINITT Healthcare Co. Ltd., Seoul, Korea). Then, the volume was calculated by multiplying the sum of the areas by the slice thickness. The accuracy of measurements by this method has been validated in previous studies ${ }^{11,21)}$. For comparison, we measured the greatest tumor diameter on two-dimensional plane at the initial and the last follow-up MRI. We analyzed the distance between tumor and optic chiasm to determine how it affects the patient outcome.

We identified the factors associated with relatively rapid tu-

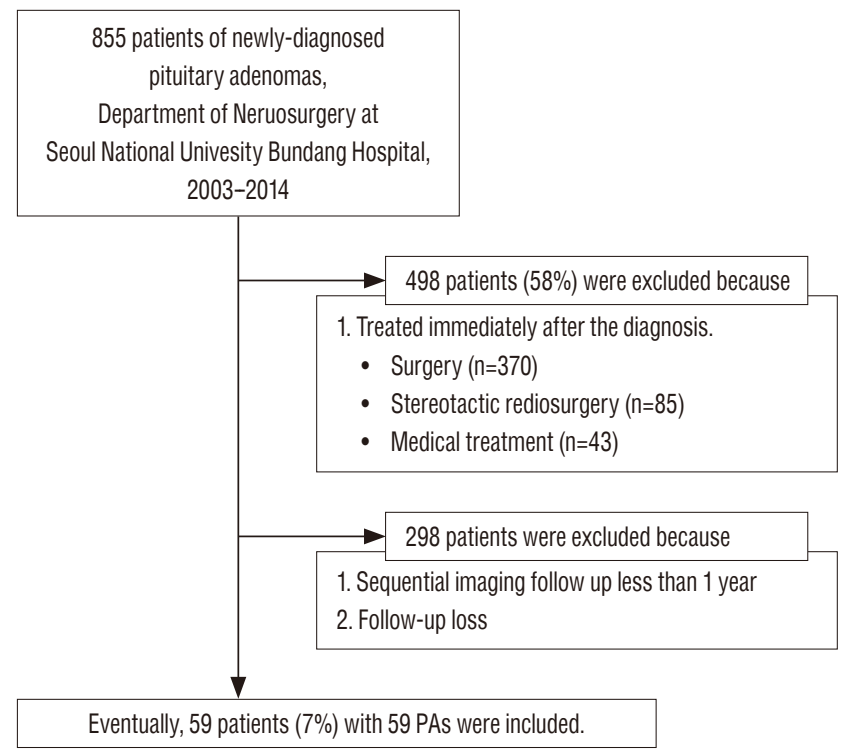

Fig. 1. Flow diagram of the patients. PAs : pituitary adenomas. 
mor growth. The definition of significant tumor growth in previous studies has shown wide variability. We believe that the rate of tumor growth is of a greater clinical importance than the extent of growth. So, we divided the PAs into the growth and stable tumor group according to the mean tumor growth rate.

\section{Statistical analysis}

All statistical analyses were performed using SPSS version 21 (IBM, Chicago, IL, USA). The categorical variables were analyzed using Pearson chi-square test and Fisher's exact test. The continuous variables were presented as the mean \pm standard deviation for parametric data. For an intergroup comparison, Student's t-test was used for parametric data and the Mann-Whitney $U$ test for non-parametric data. The cut-off value was the point closest to the point of perfect classification (sensitivity and specificity), as determined by using a receiver operating characteristic (ROC) curve with a discrimination power. Cox proportional hazards regression was used to determine the hazards ratio for the eventual treatment group.

Variables associated with a dependent variable in univariate analysis $(p<0.10)$ were then included into a multivariate analysis. A multivariate analysis was performed using logistic regression analysis with a backward stepwise method. $p$ values of less than 0.05 were considered significant.

\section{RESULTS}

\section{Characteristics of study population}

Thirty-one patients (53\%) were female. The mean age was 64.4 years and mean follow-up duration was 46.8 months. Most of tumors were incidental findings. Of the 59 patients, 47 patients (80\%) had no symptoms at all; nine patients (15\%) had clinical headache, which was not related with tumor and was controlled without any treatment; two patients (3\%) had visual symptoms, of whom only one patient had an evidence of optic nerve compression, but was reluctant to surgery; one patient $(2 \%)$ showed clinical galactorrhea, but the prolactin level was within normal range. Fifty-eight patients (98\%) had non-functioning PAs. The remaining one patients $(2 \%)$ had a suspicious prolactinoma, but was reluctant to any treatment modality including hormone therapy. So, about the reason for observation, 56 patients (95\%) had no tumor-related symp- toms or neurologic signs, two patients $(3 \%)$ were reluctant to treatment, and one patient (2\%) had poor systemic condition to undergo surgery. The demographic information and clinical features of our study population are presented in Table 1.

\section{Volumetric analysis of tumor size}

The volumetric characteristics of the 59 PAs are provided in Table 1. The mean initial tumor volume and diameter were $1.83 \pm 2.97 \mathrm{~mL}$ and $13.77 \pm 6.45 \mathrm{~mm}$, and the mean last tumor volume and diameter were $2.85 \pm 4.47 \mathrm{~mL}$ and $15.75 \pm 8.08 \mathrm{~mm}$, respectively. The mean distance between tumor and optic chiasm was $1.86 \mathrm{~mm}$ at the initial MRI and $1.32 \mathrm{~mm}$ at the last follow-up MRI. The mean annual tumor growth rate was 0.34 $\pm 0.68 \mathrm{~mL} / \mathrm{yr}$. The most appropriate cut-off point for mean tu-

Table 1. Characteristics of study population and tumor size

\begin{tabular}{|c|c|}
\hline Parameter & Value \\
\hline Total patients & 59 \\
\hline Female & $31(53)$ \\
\hline Mean age (years) & $64.4 \pm 14.6(23-83)$ \\
\hline Mean follow-up duration (months) & $46.8 \pm 32.1(12-136)$ \\
\hline \multicolumn{2}{|l|}{ Initial presentation symptoms } \\
\hline Incidental finding & $47(80)$ \\
\hline Headache & $9(15)$ \\
\hline Visual symptoms & $2(3)$ \\
\hline Galactorrhea & $1(2)$ \\
\hline \multicolumn{2}{|l|}{ Mean volume (mL) } \\
\hline Initial volume & $1.83 \pm 2.97$ \\
\hline Last volume & $2.85 \pm 4.47$ \\
\hline Mean volume change (mL) & $1.03 \pm 2.08$ \\
\hline Relative Growth rate per year (\%/yr) & $34.4 \pm 75.0$ \\
\hline Mean Growth rate per year (mL/yr) & $0.34 \pm 0.68$ \\
\hline \multicolumn{2}{|l|}{ Distribution of growth rate (mL/yr) } \\
\hline-0.3 to 0.3 & $43(73)$ \\
\hline$>0.3$ & $16(27)$ \\
\hline \multicolumn{2}{|l|}{ Mean tumor size (mm) } \\
\hline Initial tumor size & $13.77 \pm 6.45$ \\
\hline Last tumor size & $15.75 \pm 8.08$ \\
\hline \multicolumn{2}{|c|}{ Distance from tumor to optic chiasm (mm) } \\
\hline Initial MRI & $1.86(0-8.6)$ \\
\hline Last MRI & $1.32(0-7.4)$ \\
\hline
\end{tabular}

Values are presented as mean \pm standard deviation (range), number (range), or number (\%). MRI, magnetic resonance imaging 
mor growth rate using ROC curve was $0.29 \mathrm{~mL} / \mathrm{yr}$ (area under curve [AUC], 0.774; 95\% confidence interval [CI], 0.639$0.908 ; p=0.029$ ). It was almost alike with the mean tumor growth rate, so we divided the growth and stable tumor group according to the mean growth rate value of $0.3 \mathrm{~mL} / \mathrm{yr}$. Sixteen (27\%) PAs exhibited growth rate over $0.3 \mathrm{~mL} / \mathrm{yr}$ and they were classified as the growth group. The other 43 patients (73\%) were classified as the stable group.

Table 2 shows predictive factors for tumor growth. The initial tumor size (hazard ratio [HR], 1.140; 95\% CI, 1.003-1.295; $p=0.045$ ) was the only independent predictor that significantly determined the tumor growth. PAs with lobulating contour and initial tumor volume trended toward, but showed no statistical significance by multivariate analysis. No other clinical or radiological characteristics showed statistical significance.

\section{Clinico-radiological factors of clinical outcome}

After excluding three patients who needed treatment at presentation from our total population, we could determine the natural course of 56 patients with non-symptomatic nonfunctioning PAs. They showed no clinical and biochemical evidence of hormone excess at the time of diagnosis. Among 56 conservatively managed patients, six patients (11\%) with both obvious tumor growth and de novo symptom underwent surgical removal with a mean interval of $34.5 \pm 23.0$ months from diagnosis to treatment. The remaining 50 patients (89\%) were still maintained with conservative management during the follow-up periods of $48.1 \pm 30.9$ months. For the hormonal function assessment, all but one patient had no hormonal dysfunction : only one patient experienced increase of prolactin, due to stalk-resection effect as tumor grew. In the meantime, there were no pituitary apoplexy among out cohort during observation periods. Clinico-radiological characteristics of both groups are provided in Table 3 .

The whole patients of both groups were diagnosed with PAs incidentally : no one had tumor-related symptom at first. Clinical characteristics, such as age or sex, showed no significant differences between each group. There were no significant differences in volumetric characteristics : initial tumor volume, last tumor volume, or mean growth rate were not significant factors. On the contrary, the differences of initial or last tumor size in diameter, and the relationship between tumor and optic chiasm were proved to be significant between each group. The patients who eventually underwent surgery had variable tumor size and distance from tumor to optic chiasm at first. However, on last follow-up MRI, there was no interval between tumor and chiasm. The reason for surgery was due to visual symptom in all six patients : five patients had newly-developed visual field defects and one patient had aggravated visual acuity. It could be said that the patients who underwent surgery had bigger tumor size than the patients who maintain conservative management, and showed no interval between tumor and chiasm on last follow-up MRI.

We plotted the ROC curve and determined the most appropriate cut-off value for tumor diameter on last follow-up MRI. Fig. 2 shows that AUC of the tumor diameter on last follow-up MRI was 0.897 (95\% CI, 0.814-0.980, $p=0.002)$, and the cutoff value was $21.75 \mathrm{~mm}$. By Cox regression analysis, nonfunctioning PAs of last longest diameter over $21.75 \mathrm{~mm}$ were the only significant prognostic factor for eventual treatment (HR, 89.104; 95\% CI, 1.631-4867.010; $p=0.028$; Fig. 3). However, tumor diameter and distance from tumor to optic chiasm on last follow-up MRI were not significant by multivariate analysis (Table 4).

Table 2. Predictive factors for tumor growth

\begin{tabular}{|c|c|c|c|}
\hline Variable & Univariate ( $p$-value) & Multivariate ( $p$-value) & OR $(95 \% \mathrm{Cl})$ \\
\hline Sex (female) & 0.728 & & \\
\hline Age & 0.643 & & \\
\hline \multicolumn{4}{|l|}{ Radiological features } \\
\hline High T2SI & 0.896 & & \\
\hline Cystic mass (>50\%) & 0.658 & & \\
\hline Lobulating contour & 0.041 & 0.072 & $6.132(0.851-44.23)$ \\
\hline Initial tumor volume $(\mathrm{mL})$ & 0.065 & 0.081 & $1.277(0.970-1.680)$ \\
\hline Initial tumor size (mm) & 0.031 & $0.045^{*}$ & $1.140(1.003-1.295)$ \\
\hline
\end{tabular}

${ }^{*} p<0.05$. OR : odds ratio, $\mathrm{Cl}$ : confidence interval, SI : signal intensity 
Table 3. Clinico-radiological characteristics of patients who eventually underwent surgery (treatment group) and those who maintain conservative management (observation group)

\begin{tabular}{|c|c|c|c|c|}
\hline Characteristic & Total & Treatment group & Observation group & $p$-value \\
\hline No. of Lesions & 56 & $6(11)$ & $50(89)$ & \\
\hline Age at diagnosis & $63.9 \pm 14.7$ & $63.5 \pm 10.0$ & $64.0 \pm 15.2$ & 0.941 \\
\hline Male : female & $27: 29$ & $2: 4$ & $25: 25$ & 0.671 \\
\hline Intervals to surgery (months) & NA & $34.5 \pm 23.0$ & NA & \\
\hline Initial tumor volume (mL) & $1.69 \pm 2.85$ & $1.86 \pm 0.79$ & $1.68 \pm 3.00$ & 0.885 \\
\hline Last tumor volume (mL) & $2.50 \pm 3.66$ & $3.29 \pm 2.21$ & $2.40 \pm 3.81$ & 0.581 \\
\hline Mean growth rate per year (mL/yr) & $0.31 \pm 0.69$ & $0.91 \pm 1.47$ & $0.24 \pm 0.51$ & 0.319 \\
\hline Initial tumor size (mm) & $13.16 \pm 5.65$ & $18.38 \pm 4.76$ & $12.54 \pm 5.46$ & $0.015^{*}$ \\
\hline Last tumor size (mm) & $14.92 \pm 6.51$ & $22.57 \pm 2.42$ & $14.00 \pm 6.24$ & $0.000^{*}$ \\
\hline Optic chiasm compression on last f/u MRI & $6(11)$ & $3(50)$ & $3(6)$ & $0.013^{*}$ \\
\hline Initial tumor-chiasm distance (mm) & $2.32 \pm 2.04$ & $2.50 \pm 2.07$ & $0.87 \pm 0.98$ & 0.065 \\
\hline Last tumor-chiasm distance (mm) & $1.88 \pm 1.94$ & $0.0 \pm 0.0$ & $2.11 \pm 1.93$ & $0.000^{*}$ \\
\hline
\end{tabular}

Values are presented as mean \pm standard deviation or number (\%) unless otherwise indicated. ${ }^{*} p<0.05$. NA : not applicable, f/u : follow up, MRI : magnetic resonance imaging

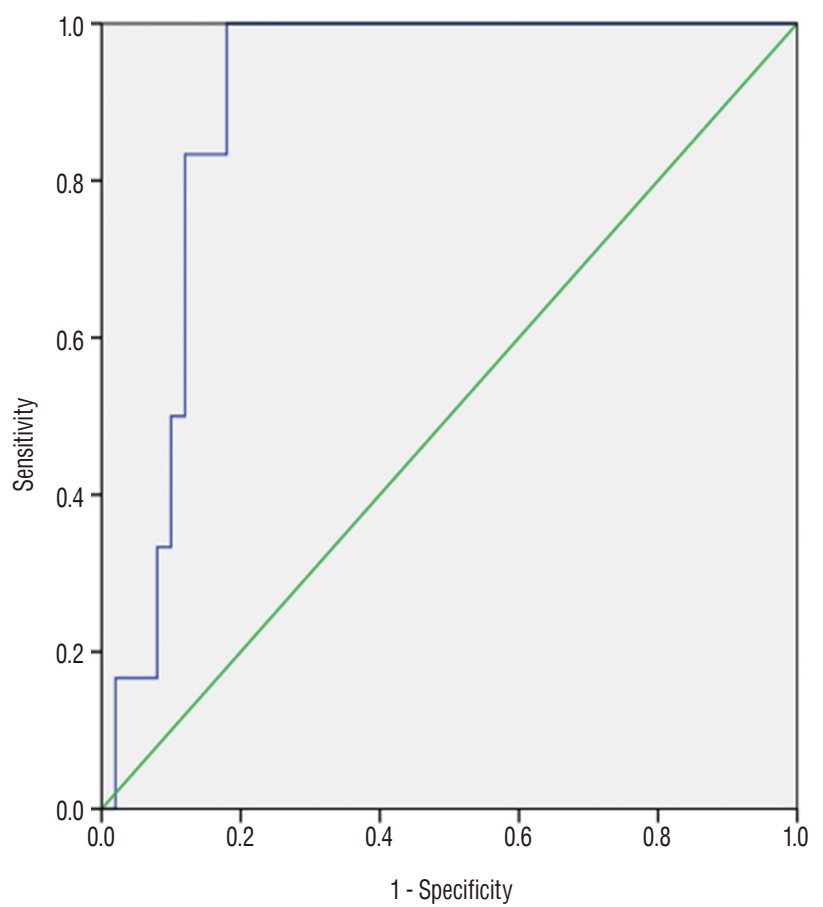

Fig. 2. Receiver operating characteristic curves for last follow-up diameter to predict eventual treatment (area under curve, 0.897; 95\% confidence interval, $0.814-0.980 ; p=0.002$ ).

\section{DISCUSSION}

Several studies have described the natural history of PAs. However, information about the tumor growth pattern and

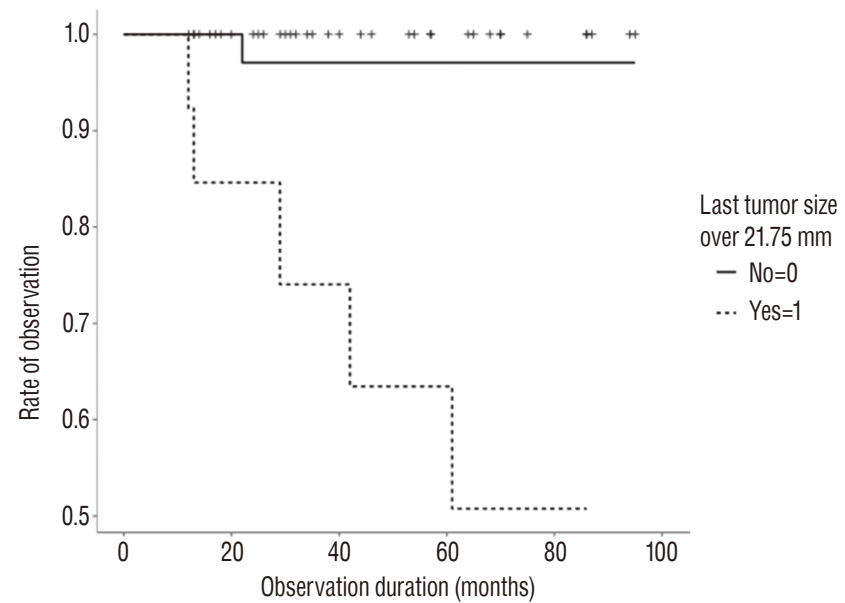

Fig. 3. By Cox regression analysis, nonfunctioning pituitary adenomas of last follow-up diameter over $21.75 \mathrm{~mm}$ were the significant prognostic factor for eventual treatment.

prognostic factors was inconclusive, because of small sample sizes and the variety of the methods used to measure the tumor growth. This study added to the literature for the following. Our analysis revealed that linear diameter was more significant to detect tumor growth rather than volume. It could be easily estimated that not the volume itself, but the longest diameter affects the optic nerve apparatus and then finally, the visual symptoms. In many previous studies, it was visual impairment that determined the eventual intervention among the conservatively managed $\mathrm{PAs}^{5}$. So, the longest diameter 
Table 4. Prognostic factors of eventual treatment among patients with a 'wait-and-see' policy

\begin{tabular}{lcc}
\hline Variable & Multivariate ( $\boldsymbol{p}$-value $)$ & OR (95\% Cl) \\
\hline Last f/u tumor diameter $(\mathrm{mm})$ & 0.120 & $0.702(0.450-1.096)$ \\
Last f/u tumor diameter $>21.75 \mathrm{~mm}$ & $0.028^{*}$ & $89.104(1.631-4867.010)^{*}$ \\
Last f/u tumor-chiasm distance $(\mathrm{mm})$ & 0.852 & \\
\hline
\end{tabular}

${ }^{*} p<0.05$. OR : odds ratio, $\mathrm{Cl}$ : confidence interval, f/u : follow up

Table 5. The natural course of tumor size in pituitary adenomas

\begin{tabular}{|c|c|c|c|c|}
\hline Study & Diagnosis & Number of patients & Mean follow-up duration & Rate of stable size (\%) \\
\hline Donovan and Corenblum ${ }^{6)}$ (1995) & $\mathrm{PI}$ & 31 & 6.4 years & 90 \\
\hline Sanno et al..$^{19)}(2003)$ & NFPA & 115 & 50.7 months & 80 \\
\hline Arita et al..2) (2006) & NFPA & 42 & $61.9 \pm 38.2$ months & 60 \\
\hline Dekkers et al. ${ }^{5)}$ (2007) & NFPA & 28 & $118 \pm 24$ months & 50 \\
\hline Anagnostis et al." (2011) & NFPA & 114 & $55 \pm 6$ months & 83 \\
\hline Fernandez-Balsells et al. ${ }^{7)}(2011)^{*}$ & PI/NFPA & 902 & 3.9 years $(3-15)$ & $\begin{array}{l}87.5 \text { in macroadenomas } \\
96.7 \text { in microadenomas }\end{array}$ \\
\hline Imran et al. ${ }^{12)}(2016)$ & PI & 328 & 3.02 years & 87 \\
\hline This study (2017) & PA & 59 & $46.8 \pm 32.1$ months & 72.9 \\
\hline
\end{tabular}

*Systematic review. PI : pituitary incidentaloma, NFPA : nonfunctioning pituitary adenoma

rather than volume determined the eventual treatment group.

Additionally, we analyzed that PAs of the longest diameter on last follow-up MRI over $21.75 \mathrm{~mm}$ were the only significant prognostic factor for eventual treatment. Several previous studies described the factors for tumor growth with PAs : macroadenomas rather than microadenomas and solid lesions $^{2,7,11,14,15,17)}$. But, there was no definite consensus to define the tumor size to determine the treatment. Moreover, to date, there was no data how big is enough to determine the eventual intervention among the conservatively managed patients. Our study suggested one possible factor. The knowledge of natural history of a disease is crucial in determining the appropriate clinical management of the patient.

In overall, the presumed nonfunctioning PAs seemed to have a small probability of tumor growth and followed a benign course ${ }^{1,2,719)}$. Also in our cohort, $73 \%$ of conservatively managed PAs remained stable in size and $11 \%$ would be likely to be treated eventually during observation periods. Table 5 summarized the literature for the natural course of tumor size in PAs. We can assume that only a minority of nonfunctioning PAs exhibit considerable growth and become clinically relevant, while the majority remain stable or show extremely slow growth velocity ${ }^{11)}$. Many previous studies have also proposed a conservative approach with careful follow-ups in patients with PAs without visual or neurological abnormali- ties ${ }^{1,6,13,18)}$. So, the 'wait-and-see' policy might seem reasonable for PAs without symptoms.

This study has several limitations. 1) As there is no concrete guideline for deciding "wait-and-see" policy for the nonfunctioning PA, so there might be any discrepancy at the decision for treatment. That is why we define concrete inclusion criteria for this study, considering its retrospective design of this study. The authors are not giving a guarantee of "wait-andsee" policy, but showing the possible fate of conservativelymanaged PAs. 2) As this study was performed by a retrospective chart review in a single institute, it could result in selection bias. 3) Only based on MRI findings, the lesions might be not PAs, but possibly be other pathologies. 4) In addition, as we analyzed two sequential imaging follow-ups on our initial impression that PAs exhibit linear growth, the serial images would reveal the exact growth modeling. And 5) the follow-up period of 12 months and longer at minimum is relatively short for tumors of naturally benign course. Further study with a longer follow-up period is a mandatory.

Despite these limitations, our study suggested new possible factor to decide the eventual treatment among patients with PAs. Additionally, our analysis revealed that linear diameter was more significant to detecting tumor growth. It would be more helpful in the clinical significance as well as in a research setting. Further serial image analyses with bigger sample size 
would be necessary.

\section{CONCLUSION}

Seventy-three percentages among PAs on a 'wait-and-see' policy showed stable tumor size during observation periods. The initial tumor size of PAs was the independent predictive factor that determined tumor growth. Six patients (11\%) of 56 conservatively managed nonfunctioning PAs were likely to be treated eventually. By Cox regression analysis, the longest diameter over $21.75 \mathrm{~mm}$ on last follow-up MRI was the significant predictive factor for eventual treatment. Further study with large series is a mandatory.

\section{CONFLICTS OF INTEREST}

No potential conflict of interest relevant to this article was reported.

\section{INFORMED CONSENT}

This type of study does not require informed consent.

\section{- Acknowledgements}

Portions of this work were presented in abstract form at the 57 th annual meeting of the Korean Neurosurgical Society, October 14, 2017.

This work was Supported by grant no 14-2016-024 from the SNUBH Research Fund.

\section{References}

1. Anagnostis P, Adamidou F, Polyzos SA, Efstathiadou Z, Panagiotou A, Kita $\mathrm{M}$ : Non-functioning pituitary adenomas: a single center experience. Exp Clin Endocrinol Diabetes 119 : 314-319, 2011

2. Arita $K$, Tominaga A, Sugiyama $K$, Eguchi $K$, lida $K$, Sumida $M$, et al. : Natural course of incidentally found nonfunctioning pituitary adenoma, with special reference to pituitary apoplexy during follow-up examination. J Neurosurg 104 : 884-891, 2006

3. Chacko AG, Chandy MJ : Incidental pituitary macroadenomas. Br J
Neurosurg 6 : 233-236, 1992

4. Costello RT : Subclinical adenoma of the pituitary gland. Am J Pathol 12 : 205-216.1, 1936

5. Dekkers OM, Hammer S, de Keizer RJ, Roelfsema F, Schutte PJ, Smit JW, et al. : The natural course of non-functioning pituitary macroadenomas. Eur J Endocrinol 156 : 217-224, 2007

6. Donovan LE, Corenblum B : The natural history of the pituitary incidentaloma. Arch Intern Med 155 : 181-183, 1995

7. Fernandez-Balsells MM, Murad MH, Barwise A, Gallegos-Orozco JF, Paul A, Lane MA, et al. : Natural history of nonfunctioning pituitary adenomas and incidentalomas: a systematic review and metaanalysis. J Clin Endocrinol Metab 96 : 905-912, 2011

8. Freda PU, Beckers AM, Katznelson L, Molitch ME, Montori VM, Post $K D$, et al. : Pituitary incidentaloma: an endocrine society clinical practice guideline. J Clin Endocrinol Metab 96 : 894-904, 2011

9. Greenman $Y$, Stern $N$ : Non-functioning pituitary adenomas. Best Pract Res Clin Endocrinol Metab 23 : 625-638, 2009

10. Hall WA, Luciano MG, Doppman JL, Patronas NJ, Oldfield EH : Pituitary magnetic resonance imaging in normal human volunteers: occult adenomas in the general population. Ann Intern Med 120 : 817-820, 1994

11. Honegger J, Zimmermann S, Psaras T, Petrick M, Mittelbronn M, Ernemann U, et al. : Growth modelling of non-functioning pituitary adenomas in patients referred for surgery. Eur J Endocrinol 158 : 287-294, 2008

12. Imran SA, Yip CE, Papneja N, Aldahmani K, Mohammad S, Imran F, et al. : Analysis and natural history of pituitary incidentalomas. Eur J Endocrinol 175 : 1-9, 2016

13. Karamouzis I, Berardelli R, Prencipe N, Berton A, Bona C, Stura G, et al. : Retrospective observational analysis of non-irradiated non-functioning pituitary adenomas. J Endocrinol Invest 38 : 1191-1197, 2015

14. Karavitaki N, Collison K, Halliday J, Byrne JV, Price P, Cudlip S, et al. : What is the natural history of nonoperated nonfunctioning pituitary adenomas? Clinical Endocrinol (0xf) 67 : 938-943, 2007

15. Lania A, Beck-Peccoz P : Pituitary incidentalomas. Best Pract Res Clin Endocrinol Metab 26 : 395-403, 2012

16. Marsden JE, Tromba AJ : Vector calculus, ed 3. New York : W H Freeman \& Co (Sd), 1988, pp241-302

17. Orija IB, Weil RJ, Hamrahian AH : Pituitary incidentaloma. Best Pract Res Clin Endocrinol Metab 26 : 47-68, 2012

18. Reincke M, Allolio B, Saeger W, Menzel J, Winkelmann W : The 'incidentaloma' of the pituitary gland. Is neurosurgery required? JAMA 263 : 2772-2776, 1990

19. Sanno N, Oyama K, Tahara S, Teramoto A, Kato Y : A survey of pituitary incidentaloma in Japan. Eur J Endocrinol 149 : 123-127, 2003

20. Teramoto A, Hirakawa K, Sanno N, Osamura Y : Incidental pituitary lesions in 1,000 unselected autopsy specimens. Radiology 193 : 161164,1994

21. Varughese JK, Wentzel-Larsen T, Vassbotn F, Moen G, Lund-Johansen $M$ : Analysis of vestibular schwannoma size in multiple dimensions: a comparative cohort study of different measurement techniques. Clin Otolaryngol 35 : 97-103, 2010 